\title{
0606 DOG BITE PREVENTION: WHAT CHILDREN KNOW
}

C A Dixon*, E M Mahabee-Gittens, C J Lindsell Correspondence: Cincinnati Children's Hospital and Medical Center, 3333 Burnet Avenue, MLC 2008 Cincinnati, Ohio 45229, USA

10.1136/ip.2010.029215.606

Background There are 4.5 million annual dog bites in the US; $>368000$ are seen in emergency departments (ED). Children ages 5-9 have the highest incidence.

Objectives To determine child dog bite prevention knowledge.

Methods Cross-sectional sample of parents and children (5-15 years), presenting to a paediatric ED. Dyads completed knowledge tests developed from Centre for Disease Control dog bite prevention recommendations. Passing score was $>70 \%$; logistic and linear regression modelled odds of passing and knowledge scores.

Results Of 300 children: mean age was 8.7 (3.1 SD), 69\% were $5-9$ years, and $51 \%$ were female. Of parents: $63 \%$ had income $>\$ 20000,57 \%$ had education $>$ high school and $51 \%$ were white. Dog ownership was $73 \%$; dog bite prevalence was $23 \%$. Over $70 \%$ of parents denied child receiving dog bite prevention education; $88 \%$ desired it. Mean child knowledge score was 10 (2.5SD). Older children had higher odds of passing (OR:1.15 (95\% CI 1.06 to 1.25$)$ ), as did children with white parents (OR:1.88 (95\% CI 1.17 to 3.02)). Children gained 0.25 score with each year of age and had 0.97 higher scores if they had white parents $(p<0.01)$. No associations were found 


\section{IP Safety 2010 abstracts}

between knowledge, socioeconomic status, dog ownership or prior dog bite.

Conclusion Dog bites are preventable child injuries. Results indicate $>40 \%$ children fail dog bite prevention knowledge testing suggesting universal dog bite prevention education is warranted, with special consideration for populations predicted to have lower dog bite prevention knowledge. 